\title{
Desarrollo de un programa psicoeducativo en inteligencia emocional para cuidadores principales de enfermos de Alzheimer
}

\author{
Esther Sitges Maciá y Beatriz Bonete López \\ Universidad Miguel Hernández de Elche (España)
}

\begin{abstract}
A comienzos del año 2000 se dieron los primeros pasos en la constatación empírica de los efectos beneficiosos de la Inteligencia Emocional (IE) sobre las personas. Sin embargo, y a pesar de estudiarse los beneficios de este constructo en diversos ámbitos como el educativo, el laboral o el de la salud, no existen demasiados trabajos centrados en el estudio que la IE pude tener sobre las habilidades sociales de los Cuidadores de Enfermos de Alzheimer. En el presente trabajo se evalúan las Habilidades Sociales y la IE de los cuidadores de enfermos de Alzheimer. Se realiza una intervención psicoeducativa a 21 cuidadores informales de enfermos de Alzheimer con un grupo experimental y un grupo control de las asociaciones de enfermos de Alzheimer de Alicante y Elche. Tras la intervención, se observa que los niveles de IE emocional en el grupo experimental se han mantenido. Los cuidadores verbalizaban una mejora tras la intervención. Creemos que los resultados son buenos como estudio preliminar y que sería interesante ampliar tanto el número de horas del taller como el de sujetos para poder generalizar los resultados obtenidos.
\end{abstract}

Palabras clave: Inteligencia Emocional, habilidades sociales, cuidadores de Alzheimer.

Development of a psychoeducational program in emotional intelligence for primary caregivers of Alzheimer patients. In early 2000 the first steps in the empirical evidence of the effects that a good emotional intelligence (EI ) can have on people, however, despite the benefits of studied this construct in various fields such as education, labor or health, there are not many studies focusing on the study of skills that EI has on caregivers of Alzheimer. In this paper Social Skills and IE caregivers of Alzheimer patients are evaluated. A psychoeducational intervention to 21 informal caregivers of Alzheimer's patients with an experimental group and a control group in the Alzheimer associations of Alicante and Elche. After surgery, it is observed that the levels of emotional IE in the experimental group were maintained. Caregivers verbalized an improvement after surgery. We believe that the results are good as a preliminary study and it would be interesting to expand both the number of hours of the workshop as the subject in order to generalize the results.

Keywords: Emotional Intelligence, social skills program, Alzheimer caregivers.

Correspondencia: Esther Sitges Maciá. Departamento de Psicología de la Salud. Universidad Miguel Hernández de Elche. Edificio Altamira. Avda. de la Universidad, s/n. C.P.: 03202. Elche, Alicante (España). E-mail: esther.sitges@umh.es 
Sabemos que el cuidado de una persona que presenta algún tipo de demencia requiere de una atención casi continua, por lo que las personas que asumen el papel de cuidador principal, que suele ser un cuidador informal, están sometidas a un notable estrés, estando por tanto estas personas en una situación de alta vulnerabilidad (Crespo y López, 2005). Además de los estados de depresión y ansiedad que puede experimentar un cuidador de un enfermo de Alzheimer (López y Crespo, 2007), también pueden darse otras emociones negativas como la tristeza, ira, soledad y/o frustración, como revelan algunos estudios sobre el duelo en estos cuidadores en relación con la pérdida de un familiar que todavía se encuentra con vida (Pérez-Godoy y Moreno-Guerín, 2007; Meuser y Marwit, 2001).

Los estudios centrados en el Estado Emocional del Cuidador, revelan que, de entre las variables que guardan una relación directa con el malestar del cuidador, se encuentra la depresión (Schulz et al., 1995; Laserna et al., 1997), la falta de apoyo social (Pushkar-Gold et al., 1995) y más concretamente la falta de satisfacción con los lazos sociales (Stuckey y Smith, 1997). En el estudio realizado por Losada y su equipo se pone de manifiesto que la angustia, el miedo y la desesperanza son los sentimientos negativos que más experimentan los cuidadores, aunque también la mayoría sentían ira y sentimiento de soledad (Losada et al., 2006).

Sabemos que para lograr un estado de bienestar personal, es necesario el manejo óptimo de las emociones (Bisquerra, 2003), por lo que para un buen desarrollo integral de la persona se pone de manifiesto la necesidad de promover y fomentar competencias emocionales y sociales (Elias et al., 1997). Sabemos que la IE engloba un conjunto de habilidades relacionadas con el procesamiento emocional de la información, en concreto, tal y como definen la IE John Mayer y Peter Salovey (1990) es "la habilidad para percibir, asimilar, comprender y regular las propias emociones y la de los demás promoviendo un crecimiento emocional e intelectual” (Mayer y Salovey, 1997).

Las investigaciones realizadas en el ámbito de las emociones en los cuidadores de enfermos de Alzheimer se han centrado, sobre todo, en depresión y ansiedad (Fernández-Hermida et al., 1990; Schulz et al., 1995). Así por ejemplo en el estudio de Muela et al. (2002) cuyo objetivo era comprobar si la relación existente entre la carga del cuidador y la ansiedad y depresión está mediada por los estilos de afrontamiento, los resultados obtenidos mostraron que las estrategias de afrontamiento centradas en las emociones hacían disminuir la ansiedad, mientras que las estrategias de afrontamiento centradas en el problema no resultaban tan eficaces. A raíz de estos resultados, los autores sugieren la necesidad de una terapia psicológica centrada en el aprendizaje de habilidades de afrontamiento dirigidas a las emociones para reducir la ansiedad de los cuidadores de personas con demencia. Concluyen, sin embargo, que se 
necesitan más estudios para evaluar las intervenciones y sus efectos a largo plazo en los cuidadores.

Por su parte, el estudio de Etxeberria-Arritxabal y colaboradores (2011) cuyo objetivo fue observar la evolución de los cuidadores de enfermos de Alzheimer que habían participado en un programa psicosocial centrado en el fomento estrategias de afrontamiento, entrenamiento en resolución de problemas, cuidado del cuidador y promoción del apoyo social durante un año, concluyeron que los cuidadores, gracias a la intervención, consiguen cambiar las atribuciones negativas que pueden tener hacia los comportamientos que tienen las personas con Alzheimer y observándose además una disminución de la sobrecarga. Así mismo, los pensamientos disfuncionales también disminuyeron en relación con el grupo control, disminuyendo por tanto el estrés relacionado a dichos pensamientos y el índice de depresión.

En el meta-análisis realizado por Brodaty et al. (2003), realizado con cuidadores familiares de pacientes con demencia, se concluyó que los programas psicoeducativos tienen efectos leves, estadísticamente significativos y positivos en el aumento de conocimiento de los cuidadores, en la morbilidad psicológica, en las estrategias de afrontamiento y en el apoyo social. También se encuentra estudios que afirman que estos programas mejoran el estado psicológico del cuidador y retrasan la institucionalización del paciente. En la misma línea, estudios más recientes han demostrado que estos programas tienen resultados positivos en reducción de la sobrecarga, la depresión y el trastorno que ocasionan las alteraciones de conducta del paciente en el cuidador. En este mismo estudio, los autores afirman que las sesiones que incluyen apoyo social y entrenamiento en diferentes habilidades son las que mayores efectos tienen en la reducción de carga y depresión (Etxebarria-Arritxabal et al., 2011).

A la vista de estas investigaciones, nos planteamos estudiar en una muestra de 21 cuidadores de enfermos de Alzheimer la influencia de las habilidades que tienen que tener los cuidadores principales de enfermos de Alzheimer, mediante una intervención de elaboración propia basada en la IE. El objetivo de la intervención es mejorar el estado emocional de los cuidadores principales de enfermos de Alzheimer, dotarlos de información, para el manejo de situaciones relacionadas con el cuidado y estrategias de afrontamiento emocional.

\section{MÉTODO}

\section{Participantes}

La muestra estaba formada por 21 cuidadores principales de enfermos de Alzheimer, pertenecientes a asociaciones de enfermos de Alzheimer de Alicante, San Vicente y Elche. Los participantes podían tener alguna relación de parentesco o no con los enfermos, pero para formar parte de la muestra los cuidadores debían identificarse a 
sí mismo como cuidador principal e informar que no se trataba de un trabajo remunerado, es decir, que se trataba de cuidadores principales informales.

\section{Variables e instrumentos}

Se elaboró un programa psicoeducativo para el aprendizaje de las habilidades de IE necesarias para un mejor manejo emocional del trabajo de cuidador informal con enfermos de Alzheimer. El programa constaba de seis sesiones basadas en los trabajos de mejora de habilidades sociales de E. Roca-Villanueva (2007).

Para la evaluación del conocimiento de los Estados Emocionales se utilizó el TMMS-24 (Trait Meta Mood Scale) de Fernández-Berrocal, Extremera y Ramos (2004), basado en Salovey, Mayer, Goldman, Turvey y Palfai (1995). El TMMS-24 contiene tres dimensiones claves de la Inteligencia Emocional con 8 ítems cada una de ellas: Atención emocional, Claridad de sentimientos y Reparación emocional. La consistencia interna informada de esta prueba es cada una de sus escalas es: percepción o atención a los sentimientos $(\alpha=0.90)$, comprensión o claridad emocional $(\alpha=0.90)$ y regulación o reparación de las emociones $(\alpha=0.86)$ (Fernández-Berrocal et al., 2004).

Para la evaluación del conocimiento de la Ansiedad, se utilizó el inventario de

Estado y Rasgo (State and Trait Anxiety Inventory-STAI) validación española (Spielberger, 1982). Es un inventario de evaluación que comprende dos escalas que miden dos conceptos de ansiedad: ansiedad-estado y ansiedad-rasgo. Mientras la ansiedad-estado se considera una condición emocional transitoria, la ansiedad-rasgo se considera como una propensión ansiosa relativamente estable (Spielbergerm, Gorsuch y Lushene, 1970).

Además, se les administró un cuestionario para obtener los datos sociodemográficos en el que se recogían la edad, el sexo, el estado civil, los estudios realizados, la situación laboral, parentesco con la persona afectada por la enfermedad, el tiempo que había transcurrido desde el diagnóstico de la demencia, el tiempo que llevaba ejerciendo de cuidador principal, si recibía alguna ayuda de otra persona para los cuidado y la cantidad de horas a la semana que ejercía de cuidador (este cuestionario se muestra en el apartado Apéndices).

Finalmente, una vez terminada la última sesión del taller, los participantes debían rellenar un último cuestionario en el que se evaluaba el propio taller. Dicho cuestionario contenía una parte con preguntas cerradas en la que los participantes contestaban de 1 a 10 a cuestiones sobre la utilidad del taller, las explicaciones dadas en el taller y la utilidad de los ejercicios realizados en el taller. También contenía una parte con preguntas abiertas en la que los participantes debían contestar qué parte del taller les gustó más, qué parte les gustó menos y qué añadirían para mejorarlo. 


\section{Procedimiento}

Para conseguir la muestra, se contactó con la Asociación de Enfermos de Alzheimer de San Vicente, Alicante y Elche (AFA). El contacto se realizó a través de la psicóloga de los centros, en primer lugar vía telefónica y posteriormente mediante una entrevista presencial con las psicólogas de los respectivos centros, para explicar la temática y el contenido de la intervención.

En un principio el estudio comenzó con 21 participantes, 14 de ellos formaban parte del grupo experimental, que pertenecían a la Asociación de Enfermos de Alzheimer de Alicante y San Vicente y 7 formaban parte de un grupo control, pertenecientes a la Asociación de Enfermos de Alzheimer de Elche. La intervención finalizó con 5 de los sujetos del grupo experimental que completaron la intervención en su totalidad y los 7 participantes del grupo control un total de 12 participantes.

La mortalidad muestral fue debida a diferentes causas personales de los participantes entre ellas, el hecho de no poder acudir por problemas físicos ya fuesen propios o de la persona con Alzheimer a su cargo. Otra razón fue la proximidad de las vacaciones de verano y viajes que algunos de los participantes tenían planeado de antemano.

El Programa llevaba por nombre "Taller para familiares: El Cuidador Asertivo”, de seis sesiones de una hora y media de duración cada una. La intervención se realizaría de forma grupal a los cuidadores principales informales de enfermos de Alzheimer. El taller fue impartido por un psicólogo con conocimientos en el tema de las habilidades sociales y la inteligencia emocional. A continuación se muestra una tabla resumen de las sesiones que tuvo el taller:

Tabla 1. Sesiones del taller

\begin{tabular}{cccccc}
\hline SESIÓN 1 & SESIÓN 2 & SESIÓN 3 & SESIÓN 4 & SESIÓN 5 & SESIÓN 6 \\
\hline $\begin{array}{c}\text { Explicación enfermedad } \\
\text { de Alzheimer y creencias } \\
\text { irracionales }\end{array}$ & $\begin{array}{c}\text { Entrenamiento } \\
\text { en relajación }\end{array}$ & $\begin{array}{c}\text { Control de } \\
\text { la ira }\end{array}$ & $\begin{array}{c}\text { Resolución de } \\
\text { conflictos }\end{array}$ & $\begin{array}{c}\text { Hacer } \\
\text { peticiones }\end{array}$ & $\begin{array}{c}\text { Afrontamiento del } \\
\text { comportamiento } \\
\text { irracional }\end{array}$ \\
\hline
\end{tabular}

Se trata de un diseño experimental de caso único $(n=5)$ con medidas pre-test y post-test. En el que mediante una intervención se intenta identificar la influencia que tienen las habilidades sociales y la inteligencia emocional en los cuidadores principales de enfermos de Alzheimer.

\section{RESULTADOS}

Los resultados estadísticos fueron obtenidos mediante el programa estadístico SPSS v.20. El perfil de cuidador corresponde a mujer (100\% en el grupo control y $85.7 \%$ en el grupo experimental) con una edad media de 57 años en el grupo control y 63 años en el grupo experimental. Casada (85.7\% control y $78.6 \%$ experimental). Con estudios 
medios finalizados (grupo control $42.9 \%$ y grupo experimental 35.7\%). Dedicándose en su mayoría a las labores del hogar (28.6\% grupo control, $35.7 \%$ grupo experimental). El parentesco corresponde al de hijo/a en el grupo control con un $71.4 \%$, mientras que en grupo experimental corresponde al de esposo/a con un 78.6\%. La media de tiempo que está diagnosticado el enfermo con Alzheimer y la media de tiempo que los cuidadores principales ejercen de dicha tarea corresponden en ambos casos con 70 meses en el grupo control y 47 meses en el grupo experimental. En el grupo control se encuentra que en su totalidad los cuidadores principales reciben ayuda por parte de otro familiar. Mientras que en el grupo experimental solo el $28 \%$ recibe algún tipo de ayuda de otro familiar. Los cuidadores dedican 125 horas semanales o más para el cuidado del enfermo de Alzheimer (grupo control 57.1\%, grupo experimental 71.4\%).

En los resultados obtenidos en el cuestionario TMMS-24 podemos observar como el grupo experimental (véase tabla 2) mantienen aproximados los niveles de atención con respecto al pre-test y al post-test (19.14 y 19.20 respectivamente). Mientras que la claridad de las emociones y la reparación de las emociones se observa una disminución de la media en el grupo post-test, con respecto al pre-test.

Tabla 2. Comparación Pre-test y Post-test, del grupo experimental (TMMS-24)

\begin{tabular}{lcc}
\hline \multicolumn{1}{c}{ Variable } & $\begin{array}{c}\text { Experimental Pre-Test } \\
\text { Media } \pm D E\end{array}$ & $\begin{array}{c}\text { Experimental Post-Test } \\
\text { Media } \pm D E\end{array}$ \\
\hline Atención de las emociones & $19.14 \pm 8.008$ & $19.20 \pm 9.884$ \\
\hline Claridad de las emociones & $27.36 \pm 9.137$ & $24.00 \pm 8.276$ \\
\hline Reparación de las emociones & $22.43 \pm 8.573$ & $21.40 \pm 3.362$ \\
\hline
\end{tabular}

En relación con el cuestionario STAI, observamos que los resultados en el grupo experimental (véase tabla 3) han aumentado tanto en Ansiedad-Estado, como en Ansiedad-Rasgo.

Tabla 3. Comparación Pre-test y Post-test del grupo experimental (STAI)

\begin{tabular}{lcc}
\hline \multicolumn{1}{c}{ Variable } & $\begin{array}{c}\text { Experimental Pre-Test } \\
\text { Media } \pm D E\end{array}$ & $\begin{array}{c}\text { Experimental Post-Test } \\
\text { Media } \pm D E\end{array}$ \\
\hline Ansiedad-Estado & $66.21 \pm 32.240$ & $92.40 \pm 6.148$ \\
\hline Ansiedad-Rasgo & $55.14 \pm 31.253$ & $74.00 \pm 8.916$ \\
\hline
\end{tabular}

Las tablas del grupo control en relación con el cuestionario TMMS-24 (véase tabla 4) muestra poca variación entre el pre-test y el post-test.

Tabla 4. Comparación Pre-test y Post-test, del grupo control (TMMS-24)

\begin{tabular}{lcc}
\hline \multicolumn{1}{c}{ Variable } & Control Pre-Test & Control Post-Test \\
& Media $\pm D E$ & Media $\pm D E$ \\
\hline Atención de las emociones & $25.29 \pm 5.880$ & $25.00 \pm 5.745$ \\
\hline Claridad de las emociones & $26.57 \pm 6.106$ & $26.29 \pm 5.880$ \\
\hline Reparación de las emociones & $27.00 \pm 4.359$ & $27.29 \pm 4.231$ \\
\hline
\end{tabular}


En el cuestionario STAI el grupo control (véase tabla 5) sigue manteniendo cierta estabilidad entre el pase previo y posterior.

\begin{tabular}{ccc}
\multicolumn{2}{c}{ Tabla 5. Comparación Pre-test y Post-test del grupo control (STAI) } \\
\hline $\begin{array}{c}\text { Control Pre-Test } \\
\text { Media } \pm D E\end{array}$ & $\begin{array}{c}\text { Control Post-Test } \\
\text { Media } \pm D E\end{array}$ \\
\hline Ansiedad-Estado & $65.00 \pm 24.987$ & $65.14 \pm 24.816$ \\
\hline Ansiedad-Rasgo & $50.14 \pm 30.482$ & $51.14 \pm 30.797$ \\
\hline
\end{tabular}

En lo referente a la percepción de los cuidadores del grupo experimental que completaron la intervención y a los que se les administró un cuestionario para que evaluaran el taller impartido. En la parte que contenía las preguntas cerradas los cuidadores puntuaban al taller con una media por encima de 9 en sus tres variables como se muestra en la tabla 6 . En relación con la parte de preguntas abiertas, todos coinciden en que, lo que no les ha gustado es la duración del taller y proponen como medida de mejora el alargar el taller con más sesiones de la misma duración.

\begin{tabular}{lc} 
Tabla 6. Percepción del taller por parte de los participantes \\
\hline \multicolumn{1}{c}{ Variables } & Media \\
\hline Utilidad para afrontar aspectos de mi vida & 9.20 \\
\hline Explicación del taller & 10 \\
\hline Utilidad de los ejercicios prácticos planteados & 9.60 \\
\hline
\end{tabular}

\section{DISCUSIÓN}

En primer lugar queremos destacar que las características sociodemográficas de nuestra muestra coinciden con las de otros estudios sobre cuidadores informales, como son que sean mujeres, de mediana edad, de estudios medios y dedicadas a las labores del hogar (Alonso et al., 2005). Los resultados obtenidos a partir de la intervención indican que no ha habido una mejora significativa en la ansiedad, sin embargo, los resultados obtenidos por el TMMS-24 (relacionado con la gestión de las emociones) se han mantenido estables. Estos resultados, aunque a primera vista puedan parecer desalentadores, creemos que son positivos si tenemos en cuenta las características de gravedad de la enfermedad de Alzheimer en la que los efectos de esta demencia neurodegenerativa se acentúan con el simple paso del tiempo, afectando de forma directa al estado psicológico del cuidador. Como se indica en el estudio de Etxeberria-Arritxabal et al. (2005), el comportamiento de los enfermos de Alzheimer afecta a los pensamientos disfuncionales de los cuidadores, lo que tiene relación con la sobrecarga que estos padecen.

Creemos también que la diferencia de puntuaciones obtenidas entre el grupo control y el grupo experimental, aunque no sean estadísticamente significativas, se observaba ya en el análisis pre-test, obteniendo el primero mejores puntuaciones en su 
estado psicológico y una mejor gestión de las emociones. Quizá el hecho de que el grupo control pertenezca a una asociación diferente puede estar influenciando en dichos datos. Así mismo, es importante destacar, el dato de que el $100 \%$ de los sujetos del grupo control afirma tener apoyo por parte de otro familiar o cuidador para hacerse cargo de la persona con Alzheimer, mientras que para el grupo experimental sólo el $28 \%$ afirma tener algún tipo de ayuda. Sabemos que éste es un aspecto fundamental pues, tal y como afirman Schneiner et al. (1999), la baja tasa de ayuda informal es un factor de estrés para los cuidadores de pacientes dementes y una gran cantidad de ayuda informal es factor protector contra el estrés.

A pesar de que los resultados no indican que la intervención haya tenido beneficios objetivos en el control de las emociones y en las habilidades sociales de éstos, los asistentes verbalizaban cualitativamente una mejora, dado que apuntaron que se sentían más capaces de hacer frente el día a día de los cuidados que requiere la persona que está a su cargo y que el taller impartido y los temas tratados en él, les fueron de utilidad, tal y como muestran los datos obtenidos en los cuestionarios que evaluaban la valoración del taller impartido. Nuestros resultados coinciden con el estudio, por ejemplo de Etxeberria-Arritxabal et al. (2005), en el que se destaca que la opinión subjetiva de los sujetos que participaron en la intervención difería con los datos objetivos. Creemos que es importante destacar que, a pesar de que la intervención estaba centrada en aportar conocimientos teóricos y prácticos sobre las habilidades sociales y la inteligencia emocional, los participantes valoraron muy positivamente el hecho de poder tener esa hora y media que duraba la sesión, para el desahogo emocional, además de poder compartir experiencias propias con personas que se encuentran en situaciones similares. Lo que nos vuelve a sugerir la importancia que tiene el apoyo social en los cuidadores además de la importancia de los grupos de ayuda (Molina et al., 2005).

Para finalizar nos gustaría concluir que, por parte de las asociaciones valoren la importancia no sólo de tratar a los pacientes con la enfermedad, sino también valorar el ofrecer apoyo y ayuda a las familias y cuidadores de los enfermos de Alzhéimer por parte de la asociación. Como conclusión final valoramos muy positivamente los resultados obtenidos en este estudio como un primer acercamiento a la intervención en IE en cuidadores de enfermos de Alzheimer y creemos que, para una generalización de los datos obtenidos, sería necesaria replicarlo con una muestra mayor la muestra y quizás realizar un programa de entrenamiento en HHSS un poco más amplio, tal y como solicitaban los participantes.

\section{REFERENCIAS}

Alonso-Babarro, A. Garrido-Barral, A., Matín-Martínez, M.A. y Francisco-Morejor, N. (2005). Evaluación de una intervención en cuidadores de pacientes con demencia (progama 
ALOIS) mediante una escala de calidad de vida. Revista Española de Geriatría y Gerontología, 40, 40-45.

Bless, H. (2000). The interplay of affect and cognition: the mediating role of general knowledge structures. En J.P. Forgas (Ed.), Feeling and thinking. The role of affect in social cognition (pp. 201-222). Cambridge: Cambridge University Press.

Brodaty, H., Green, A. y Koschera, A. (2003). Meta-Analysis of psychological interventions for caregivers of people with dementia. J Am Geriatr Soc. 51, 657-664.

Cano-Vindel, A. y Miguel-Tobal, J.J. (2001). Emociones y salud. Ansiedad y Estrés, 7(2-3), 11121.

Crespo-López, M. y López-Martínez, J. (2005). Estrés y estado emocional en cuidadores de familiares mayores con y sin demencia. Revista Española de Geriatría y Gerontología, 40, 55-61.

Elias, M.J., Zins, J.E., Weissberg, R.P., Frey, K.S., Greenberg, M.T., Haynes, N.M., Kessler, R., Schwab-Stone, M.E. y Shriver, T.P. (1997). Promoting social and emotional learning: Guidelines for educators. Alexandria. VA: Association for Supervision and Curriculum Development.

Etxeberría-Arritxabal, I., García, A., Iglesias, A., Urdaneta, E., Lorea, I., Díaz, P. y Yanguas, J.J. (2011). Efectos del entrenamiento en estrategias de regulación emocional en el bienestar de cuidadores de enfermos de Alzheimer. Revista Española de Geriatría y Gerontología, 46(4), 206-212.

Extremera, N. y Fernández-Berrocal, P. (2005). Inteligencia emocional y diferencias individuales en el metaconocimiento de los estados emocionales: una revisión de los estudios con el Trait Meta-Mood Scale. Ansiedad y Estrés, 11, 101-122.

Fernández-Berrocal, P., Extremera, M. y Ramos, N. (2004). Validity and reliability of the Spanish modified version of the Trait Meta-Mood Scale. Psychological Reports, 94, 751-755.

Fernández-Berrocal, P. y Ramos, N. (2005). Evaluando la inteligencia emocional. En P. Fernández-Berrocal y N. Ramos (Eds.), Corazones Inteligentes (pp.35-38). Barcelona: Kairós.

Fernández-Berrocal, P. y Extremera, N. (2007). Inteligencia emocional y salud. En Mestre, J.M. y Fernández-Berrocal, P. (Eds), Manual de Inteligencia emocional (pp. 173- 187). Madrid: Ed. Pirámide.

Limonero, J., Tomás-Sábado, J., Fernández-Castro, J. y Gómez-Benito, J. (2004). Influencia de la inteligencia emocional percibida en el estrés laboral de enfermería. Ansiedad y Estrés, 10(1), 29-41.

Lizeretti, N.P., Oberst, U., Chamarro, A. y Farriols, N. (2006). Evaluación de la Inteligencia Emocional en pacientes con psicopatología: resultados preliminares usando el TMMS-24 y el MSCEIT. Ansiedad y Estrés, 12(2-3), 355-364.

Losada, A., Montorio, I., Izal, M., Márquez, M. (2006). Estudio e intervención sobre el malestar psicológico de los cuidadores de personas con demencia. El papel de los pensamientos disfuncionales. Madrid: IMSERSO. Colección Estudios Serie Dependencia, No 12005.

Martos, A.J. y Gómez, M.A. (2004). Análisis de la inteligencia emocional en un grupo de personas mayores. Geriátrika, 20(6), 256-263.

Meuser T. y Marwit S. (2001). A comprenhensive, stage sensitive model of grief in dementia caregiving. Gerontologist, 41, 658-670.

Molina-Linde, J.M., Iáñez-Velasco, M.A. y Iáñez-Velasco B. (2005). El apoyo social como modulador de la carga del cuidador de enfermos de Alzheimer. Psicología y Salud, 15, 33-43. 
Muela-Martínez, J., Torres-Colmenero, C. y Peláez-Peláez, E. (2002). Comparación entre distintas clasificaciones de las estrategias de afrontamiento en cuidadores de enfermos de Alzheimer. Psicothema, 14, 558-563.

Ortega-Navas, M. (2010). La educación emocional y sus implicaciones en la salud. REOP, 21, 462-470.

Paveza, G., Cohen, D., Eisdorfer, C., Freels, S., Semla, T., Asford, W., Gorelick, P., Hirschman, R., Luchins, D. y Levy, P. (1992). Severe family violence and Alzheimer's disease: Prevalence and risk factors. The Gerontologist. 32, 492-497.

Payne, R. (2005). Técnicas de relajación: Guía práctica (4 $4^{\mathrm{a}}$ ed). Badalona: Paidotribo.

Peinado-Portero, A. y Garcés de los Fayos-Ruiz, E. (1998). Burnout en cuidadores principales de pacientes con Alzheimer: El síndrome del asistente desasistido. Anales de Psicología, 14, 83-93.

Pérez, N. y Castejón, J.L. (2007). La inteligencia emocional como predictor del rendimiento académico en estudiantes universitarios. Ansiedad y Estrés, 13(1), 119-129.

Pérez-Godoy, E. y Moreno-Geruín, A. (2007). Duelo y muerte en cuidadores familiares y profesionales de enfermos de Alzheimer. Gerokomos, 18(2), 68-71.

Pushkar-Gold, D., Cohen, C., Shulman, K., Zucchero, C., Andres, D. y Etezadi, J. (1995). Caregiving and dementia. International Journal of Aging and Human Development, 41, 183-201.

Roca-Villanueva, E. (2007). Cómo mejorar tus habilidades sociales. Programa de asertividad autoestima e inteligencia emocional. Valencia: ACDE Psicología.

Rodríguez-Marín, J. (1995). Promoción de la salud y prevención de la enfermedad: Estilos de vida y salud. Psicología Social de la Salud. Madrid: Ed. Síntesis.

Sanders, S., Ott, C., Kelberg, S. y Noonan, P. (2008). The experience of high levels of grief in caregivers of persons with Alzheimer's disease and related demential. Death stud, 32(6), 495-523.

Schulz, R., O’Brien, A.T., Bookwala, J. y Fleissner, K. (1995). Psychiatric and physical morbidity effects of dementia caregiving. Prevalence, correlates, and causes. The Gerontologist. 35,771-791.

Schneider, J., Murria, J., Benerjee, S. y Mann, A. (1999). EUROCARE: A cross-national study of co-resident spouse carers for people with Alzheimer's disease: I. Factor associated with cared burden. International Journal of Geriatric Psychiatry. 14, 651-661.

Spielberger C., Gorsuch, R. y Lushene, R. (1970). The State-Trait anxiety inventory. Palo Alto, California: Cosulting Psychologist Press.

Spielberger, C., Gorsouch, R. y Lushene, R. (1982). STAI Manual for the State-Trait Anxiety Inventory (Self Evaluation Questionaire) Madrid: TEA Ediciones S.A.

Stuckey, J. y Smyth, K. (1997). The impact of social resources on tha Alzheimer's disease caregiving experience. Research on Aging, 19, 423-441.

Vázquez-Sánchez, M., Aguilar-Trujillo, M., Estébanez-Carvajal, F., Casals-Vázquez, C., Casals-Sánchez, J. y Heras-Pérez, M. (2012). Influencia de los pensamientos disfuncionales en la sobrecarga de los cuidadores de personas dependientes. Enfermería Clínica, 22, 11-7.

Weisinger, H. (2001). La inteligencia emocional en el trabajo. Madrid: Suma de letras.

Recibido: 29 de abril de 2014

Recepción Modificaciones: 26 de mayo de 2014

Aceptado: 11 de agosto de 2014 